\title{
Micrometastasis in lymph node
}

\author{
S. Masood \\ Department of Pathology, University of Florida, HSC, Jacksonville, USA
}

\begin{abstract}
Determining whether cancer has spread to the lymph nodes is important in predicting survival from breast cancer and determining what treatment a patient requires. The traditional method of detecting such metastases, complete axillary node dissection, can result in lymphedema and other quality-of-life damaging side effects. The new technique of sentinel lymph node biopsy (SLNB) offers a way to test for lymph node metastases without causing the side effects of traditional axillary node dissection. Intraoperative analysis of SLNB is becoming an effective tool in assessment of the presence or absence of metastatic tumor, and therefore influences the treatment offered to the patient. Because of this central diagnostic use, as it is a new procedure, further studies need to be conducted to fully assess its role in breast cancer treatment.
\end{abstract}

Keywords: Breast cancer; Micrometastasis; Sentinel lymph node biopsy; Sentinel node

\section{Article}

Lymph node metastasis is the most powerful predictor of survival in breast cancer patients. Complete axillary node dissection has been the traditional approach to assess the presence or absence of lymph node metastasis. This procedure however may result in lymphedema and other discomforts to patients such as damage to nerve and blood vessels, postoperative pain and limited arm mobility. The incidence of lymphedema varies greatly depending on its clinical presentation, patient characteristics, measurement techniques and the length of follow up. Lymphatic mapping and sentinel lymph node biopsy (SLNB) is a relatively new procedure that provides accurate nodal staging without the morbidity commonly associated with total axillary node dissection.

Correspondence to: S. Masood MD, Professor \& Associate Chair, Department of Pathology, 655 W. 8th St, Jacksonville, FL 32209, University of Florida, USA. E-mail: shahla.masood@jax.ufl.edu; Tel: +1 904244 4387; Fax: +1904244 4060 .

Received: 16/05/05

Accepted: 08/09/05

First published online 29/03/06

BCO/334/2005/FO
For example, the lymphedema rate after SLNB ranges from $1 \%$ to $2 \%$ vs. $10 \%$ to $30 \%$ after axillary lymph node dissection.

It appears that SLNB is rapidly emerging as a new standard of care for management of patients with breast cancer. Currently, SLNB has replaced the formal axillary node dissection in a certain percentage of patients with $\mathrm{T} 1$ and $\mathrm{T} 2$ clinically node negative breast cancers. Studies have demonstrated that SLNB is highly predictive of axillary nodal status. The false negative rate of SNLB is reportedly $<10 \%$. However, adopting SNLB in routine clinical practice requires clear understanding of the implicit limitations of this procedure in clinical practice. SLNB requires qualified individuals in nuclear medicine, pathology, and surgery with the ability to adapt to the recommended guidelines offered by the scientific community.

Training in the technique of SLNB is a critical step in reducing the number of false negatives. If SLNB is to replace total axillary node dissection it must accurately represent the status of the entire axillary lymph nodes. It is suggested that a surgeon must achieve a detection rate of $>90 \%$ and a false negative rate of $<5 \%$ before he/she can abandon performing a concomitant traditional axillary node dissection. 
SLNB has brought a new dimension to the importance of detection of micrometastasis in breast cancer patients. Since SLNB removes only those nodes that are presumed to be the most important to the diagnosis of metastatic tumor and their numbers are usually limited to only one to three nodes, new surgical pathology protocols have emerged. These procedures include multilevel sectioning, cytokeratin immunocytochemistry and possible reverse transcriptasepolymerase chain reaction (RT-PCR) analysis of SLNB specimen.

Intraoperative analysis of SLNB is becoming an effective tool in assessment of the presence or absence of metastatic tumor. This exercise will expedite staging and potentially obviate the need for additional surgical procedures. The decision to perform imprint cytology vs. frozen section should be based on the level of experience of a pathologist in cytopathology. Smaller lymph nodes are difficult to assess by frozen section since a significant proportion of tissue may be lost during the frozen section procedure. It is desirable to achieve up to $90 \%$ accuracy between intraoperative pathologic analysis of SLNB compared to permanent sections. The Association of Directors of Surgical Pathology recommends that an intraoperative analysis should only be performed if an immediate therapeutic discussion will be made and acted upon based on the results. The College of American Pathologists consensus suggests a thorough gross examination and states that imprint cytology is preferable due to its tissue conservation relative to cryostat sectioning.

The panel of physicians participating in the 2001 Philadelphia Consensus Conference has endorsed this position. Suggestions have also been made to sectioning of the nodes at 2-mm intervals and to perform three 'levels'. However, in clinical practice there is significant variability in specimen handling and tissue processing of SLNB. Nevertheless, in order to detect micrometastatic tumor defined as $2-\mathrm{mm}$ deposit, it is reasonable to assume that sentinel lymph nodes should be sectioned at 2-mm intervals. Others have suggested a combination of frozen section and imprint cytology for an intraoperative assistant of SNLB.

Currently, there are no fully agreed upon scientific evidence to justify cytokeratin immunostaining for SLNB in routine clinical practice. It is not yet clear what is the full impact of the 'isolated tumor cells' relative to overall survival. In addition, there are still controversies regarding the prognostic significance of detection of micrometastasis in breast cancer.

Similarly, there is no scientific evidence that clusters of malignant tumor cells equal or less than $0.2 \mathrm{~mm}$ in diameter, that is 'submicrometastasis' predict an unfavorable outcome. Interestingly, a suggestion has been made that an immunostained positive
SLNB may be related to prior biopsy. A recent study from Memorial Sloan-Kettering Cancer Center demonstrated that H\&E stained positivity of SLNB was associated with tumor size, type, location, and presence of lymphovascular invasion. However, immunostained positive SLNB was not associated with any of the above-mentioned parameters, but was associated with method of biopsy. The authors in this study suggest that some of the immunostained positive SNLB is due to displaced epithelium rather that true metastasis. Therefore caution has to be exercised in interpreting these findings.

Ideally, pathologists should have access to the original core or equivalent samples of primary breast tumor before interpretation of SLNB. This analysis will provide familiarity with the histologic appearance and biologic behavior of the lesion. Suggestions are made that patients with favorable breast cancer histology have a small risk of axillary SLNB metastasis. These histologic subtypes include tubular, mucinous, papillary and medullary carcinomas. SLNB may identify a small subgroup of patients with the above mentioned favorable pathology group who may have positive metastatic tumor and may benefit from adjuvant chemotherapy. The decision to perform SLNB in patients with favorable breast cancer should take into account the $4 \%$ incidence of lymph node metastasis and the risk and benefits associated with SLNB on an individual basis.

The use of SLNB in patients with ductal carcinoma in situ is also controversial because of the low morbidity of SLNB and the small but significant rate of metastatic disease in ductal carcinoma in situ patients; it is advisable to identify patients who may benefit from this practice. SLNB may be justifiable in patients with palpable and mammographic mass, those with histologically high nuclear grade or high grade with necrosis and those with multicentric disease requiring mastectomy. The histologic underestimation of an invasive disease by core needle biopsy may also justify performing SLNB in patients with ductal carcinoma in situ diagnosed by core needle biopsy.

In summary, in the last several years enhanced public awareness, advanced technology in breast imaging and molecular genetic testing have resulted in earlier breast cancer detection. In addition, minimally invasive diagnostic and therapeutic procedures and molecular targeted therapy have brought significant change in the fundamental delivery of breast health care. These changes have brought unique challenges to pathologists, radiologists, surgeons, oncologists, researchers, and above all the patients. To overcome these challenges, attempts should be made to balance between the enthusiasm of adapting new procedures and the wisdom behind adhering to the scientifically sound measures. SLNB is 
just another minimally invasive procedure, which has a great deal of potentiality to minimize the cost and the discomfort associated with axillary node sampling. There are however, many questions, which are waiting for an answer. Perhaps the current and ongoing clinical trials will bring some light. Long term follow up studies will yield more accurate assessment of prognostic significance of SLNB and its value in the prevention of breast cancer related morbidity and its prognostic significance.

\section{Further reading}

1. Association of Directors of Anatomic Surgical Pathology. ADASP recommendations for processing and reporting lymph node specimens submitted for evaluation of metastatic disease. Am J Surg Pathol 2001; 25: 961-963.

2. Cady B. Consensus on sentinel node biopsy. Breast $J$ 2002; 8: 123-125.

3. Mittendorf ME, Arciero CA, Gutchell V, et al. Core biopsy diagnosis of ductal carcinoma in situ: an indication for sentinel lymph node biopsy. Curr Surg 2005; 62: 253-257.

4. Fitzgibbons PL, Page DL, Weaver D, et al. Prognostic factors in breast cancer. Arch Pathol Lab Med 2000; 124: 966-978.

5. Golshan M, Martin WJ, Dowlatshahi K. Sentinel lymph node biopsy lowers the rate of lymphedema when compared with standard axillary lymph node dissection. Am J Surg 2003; 69: 209-211.

6. Intra M, Veronesi P, Mazzarol G, et al. Axillary sentinel lymph node biopsy in patients with pure ductal carcinoma in situ of the breast. Arch Surg 2003; 138: 309-313.

7. Kelly TA, Kim JA, Patrick R, et al. Axillary lymph node metastases in patients with a final diagnosis of ductal carcinoma-in-situ. Am J Surg 2003; 186: 368-370.

8. Klauber-DeMore N, Tan LK, Liberman L, et al. Sentinel lymph node biopsy: is it indicated in patients with high-risk ductal carcinoma-in-situ and ductal carcinoma-in-situ with microinvasion? Ann Surg Oncol 2000; 7: 636-664.

9. Krag D, Weaver D, Ashikaga T, et al. The sentinel node in breast cancer - a multicenter validation study. N Eng J Med 1998; 339: 941-946.

10. Krag DN, Harlow S. Current status of sentinel node surgery in breast cancer. Oncology (Huntingt) 2003; 17: 1663-1666.

11. McMasters KM, Wong SL, Chao C, et al. Defining the optimal surgeon experience for breast cancer sentinel lymph node biopsy: a model for implementation of new surgical techniques. Ann Surg 2001; 234: 292-299.

12. Mendez J, Fey J, Cody H, et al. Can sentinel lymph node biopsy be omitted in patients with favorable breast cancer histology? Ann Surg Oncol 2004; 12: 24-28.

13. Moore K, Thaler H, Tan L, et al. Immunohistochemically detected tumor cells in the sentinel lymph nodes of patients with breast carcinoma: biologic metastasis or procedural artifact? Cancer 2004; 100: 929-934. 